\title{
Modeling apical columnar epithelium mechanics from circumferential contractile fibres
}

\author{
A. R. B. Boyd · S. Moore · J. E. Sader · P. V. S. Lee
}

Received: date / Accepted: date

\begin{abstract}
Simple columnar epithelia are formed by individual epithelial cells connecting together to form single cell high sheets. They are a main component of many important body tissues, and are heavily involved in both normal and cancerous cell activity. Prior experimental observations have identified a series of contractile fibres around the circumference of a cross section located in the upper (apical) region of each cell. While other potential mechanisms have been identified in both the experimental and theoretical literature, these circumferential fibres are considered to be the most likely mechanism controlling movement of this cross section. Here we investigated the impact of circumferential contractile fibres on movement of the cross section by creating an alternate model where movement is driven from circumferential contractile fibres, without any other potential mechanisms. In this model, we: utilised a circumferential contractile fibre representation based on investigations into the movement of contractile fibres as an individual system, treated circumferential fibres as
\end{abstract}

\section{A. R. B. Boyd}

Department of Mechanical Engineering, University of Melbourne, Victoria, 3010, Australia

\section{S. Moore}

IBM Research Australia, Level 5, 204 Lygon Street, Carlton, Victoria, 3010, Australia

\section{J. E. Sader}

School of Mathematics and Statistics, University of Melbourne, Victoria, 3010, Australia

P. V. S. Lee

Department of Mechanical Engineering, University of Melbourne, Victoria, 3010, Australia

E-mail: pvlee@unimelb.edu.au a series of units, and matched our model simulation to experimental geometries. By testing against laser ablation datasets sourced from existing literature, we found that circumferential fibres can reproduce the majority of cross section movements. We also investigated model predictions related to various aspects of cross section movement, providing insights into epithelium mechanics, and demonstrating the usefulness of our modeling approach.

Keywords Columnar Epithelium · Laser Ablation . Mechanistic Modeling · Vertex Model · Computational Biomechanics · Drosophila

\section{Introduction}

Simple epithelia are formed by individual epithelial cells combining into a single cell high sheet. They are categorised by the shape of the individual cells, namely flat (height less than width), cuboidal (height equal to width) or columnar (height greater than width) epithelia. Each cell within an epithelium generally has a cross section located near the top (apical region) of the cell that undergoes some form of contractile based movement (Fig. 1) (Fristrom and Fristrom, 1975; Drenckhahn and Dermietzel, 1988; Major and Irvine, 2005; Smutny et al, 2010; Ebrahim et al, 2013). This cross section has a variety of different names throughout the literature, but herein is referred to as the contractile cross section (CCS). The CCS produces forces that help hold epithelial cells together, and also plays a central role in movement of the epithelium and its constituent cells (Farhadifar et al, 2007; Liu and Cheney, 2012). The movement of the simple columnar epithelium is important for many body tissues, including the stomach and intestines (Drenckhahn and Dermietzel, 1988; 
Wang et al, 1996), and is involved in both normal and cancerous activity. Understanding the forces and movement of the columnar epithelium CCS can provide insight into the movement of these tissues, and could help identify new therapeutic approaches for cancer.

In the 1970 s and 1980 s, the ring of contractile fibres surrounding the circumference of the CCS was identified (Mooseker, 1985) and found to have strong contractile properties (Owaribe et al, 1981; Burgess, 1982; Keller et al, 1985). These experimental investigations, and theoretical work in the early 90s (Weliky and Oster, 1990), indicated that epithelial circumferential contractile fibres were the key mechanism driving the majority of epithelial sheet processes (Davidson, 2012). Recently, however, new experimental investigations have suggested that other cell mechanisms exist and may play a part sheet movement (Gorfinkiel and Blanchard, 2011; Mao and Baum, 2015). The main work for individual epithelial cells was completed by Wu et al (2014), who found contraction not related to circumferential fibres throughout the top (apical) and side (lateral) regions of individual cells.

More recent experiments have utilised laser ablation to investigate many different aspects of epithelial cells (Farhadifar et al, 2007; Bonnet et al, 2012; Louveaux et al, 2016). A common experiment uses a laser to sever the adjacent circumferential contractile fibres along the edge of two cells (Fig. 4) (Farhadifar et al, 2007; Rauzi et al, 2008; Ma et al, 2009) causing the columnar epithelium to relax. This sheet relaxation is often considered to give insight into the tension of the severed fibre prior to ablation (Landsberg et al, 2009; Wu et al, 2014), and is also commonly used as a validation technique for theoretical models (Farhadifar et al, 2007; Hutson et al, 2009).

Theoretical approaches give insight into the mechanisms controlling the mechanics of specific epithelia, and can also be used to investigate different aspects of the epithelium, such as cell patterning (Farhadifar et al, 2007). For a review of epithelium modeling refer to Brodland (2004). The current approaches to modeling epithelium movement can be categorised as vertex (Weliky and Oster, 1990; Weliky et al, 1991; Nagai and Honda, 2001; Farhadifar et al, 2007; Hufnagel et al, 2007; Hilgenfeldt et al, 2008; Honda et al, 2008b; Landsberg et al, 2009; Aegerter-Wilmsen et al, 2010; Staple et al, 2010; Mao et al, 2011; Schilling et al, 2011; Wartlick et al, 2011; Aliee et al, 2012; Salbreux et al, 2012; Smith et al, 2012; Osterfield et al, 2013; Spahn and Reuter, 2013; Du et al, 2014; Fletcher et al, 2014; Bambardekar et al, 2015; Rauzi et al, 2008; Collinet et al, 2015; Tetley et al, 2016), cell centre (Graner and Sawada, 1993; Meineke et al, 2001; Li and Sun, 2014) or sub cellular lattice (also called cellular Potts) (Graner and Glazier, 1992; Glazier and Graner, 1993; Mombach et al, 1993; Noppe et al, 2015). Vertex models describe the boundary of cells as a series of points (vertices) connected by straight segments (edges), where a vertex can be defined as a point where three or more cells are touching (Weliky and Oster, 1990). Forces are applied to the vertex points, causing them to move, and therefore controlling the shape of the cells in the epithelium. There is also a series of models that combine a vertex approach with finite element modeling (Brodland and Clausi, 1994; Chen and Brodland, 2000, 2008; Hutson et al, 2009), and a number of three dimensional implementations of vertex modeling (Murisic et al, 2015; Osterfield et al, 2013; Honda et al, 2008a,b, 2004; Okuda et al, 2013; Hannezo et al, 2014; Bielmeier et al, 2016).

Regardless of category, each model is driven by a description of either the energy or forces of the epithelium. These descriptions determine how the cells move, and is developed based on the underlying mechanisms controlling the movement of the epithelium. Theoretical models generally focus on either columnar epithelia (Farhadifar et al, 2007; Hilgenfeldt et al, 2008; Landsberg et al, 2009; Aegerter-Wilmsen et al, 2010; Schilling et al, 2011; Osterfield et al, 2013; Li and Sun, 2014; Noppe et al, 2015) or the flat epithelium that occurs during Drosophila dorsal closure (amnioserosa epithelium) (Rauzi et al, 2008; Hutson et al, 2009; Ma et al, 2009; Solon et al, 2009; Jayasinghe et al, 2013). While the flat amnioserosa epithelium does have a series of circumferential fibres, it also exhibits pulse like motions (Solon et al, 2009) and has a contractile fibre meshwork throughout the CCS of each cell (Rauzi and Lenne, 2011). Columnar epithelia do not exhibit pulse like motions, nor the contractile fibre meshwork (Drenckhahn and Dermietzel, 1988), and therefore have both different epithelium scale movement and different underlying mechanisms of movement to the flat epithelium of the amnioserosa.

The movement of the CCS of the columnar epithelium is commonly described based on three types of mechanisms: cell geometry constraints, line tension, and cell perimeter contractility. Various interpretations of these mechanisms are used, which have exceptions, and may not all be present in an individual model. Cell geometry constraints are mainly individual cell conservation of volume (Hufnagel et al, 2007), individual cell CCS conservation of area (Hilgenfeldt et al, 2008) and cell area elasticity (Farhadifar et al, 2007; Landsberg et al, 2009; Staple et al, 2010; Mao et al, 2011; Salbreux et al, 2012; Smith et al, 2012; Li and Sun, 2014; Rauzi et al, 2008; Collinet et al, 2015; Tetley et al, 2016). 
Cell area elasticity is where cell conservation of volume causes a resistance of the CCS of each cell to changes in area. This resistance is calculated from the difference between each cell's area and a reference area. The reference area is generally considered to be the same for all cells in the epithelium.

Line tension (Nagai and Honda, 2001; Farhadifar et al, 2007; Hilgenfeldt et al, 2008; Landsberg et al, 2009; Staple et al, 2010; Mao et al, 2011; Salbreux et al, 2012; Rauzi et al, 2008; Collinet et al, 2015; Tetley et al, 2016) generally represents any forces that arise from cell to cell interactions along the cell boundaries. For energy descriptions, line tension is mainly treated as proportional to the length of each cell edge (Farhadifar et al, 2007), while force descriptions generally represent it as a constant force applied along cell edges (Hutson et al, 2009). Line tension is generally considered to include forces from both circumferential contractile fibres and cell-cell adhesion (Brodland, 2002).

Cell perimeter contractility generally represents contractile forces created by the circumferential contractile fibres, where the fibres are considered to be one band surrounding the edges of each cell (Farhadifar et al, 2007). In energy descriptions, contraction of this band is treated as either proportional to cell perimeter squared (Farhadifar et al, 2007; Landsberg et al, 2009; Aegerter-Wilmsen et al, 2010; Staple et al, 2010; Mao et al, 2011; Wartlick et al, 2011; Aliee et al, 2012; Li and Sun, 2014; Noppe et al, 2015; Tetley et al, 2016) or perimeter extension relative to a reference minimum perimeter length (Hufnagel et al, 2007; Hilgenfeldt et al, 2008; Rauzi et al, 2008; Collinet et al, 2015). This reference length is considered to be the same for all cells. In a force description, Smith et al (2012) also treated band contraction as directly proportional to cell perimeter.

In the flat epitheilum, Solon et al (2009) and Jayasinghe et al (2013) represented contractile fibres with a series of springs connected both along cell edges and from cell edges to the cell centre. The forces of these springs were determined by their displacement relative to a preferred resting length that was the same for all springs throughout the epithelium. Additionally each spring had a representation of contractile movement that was either oscillatory or tension based. Also, Hutson et al (2009) utilised viscoelastic descriptions of contractile components of the amnioserosa, via either circumferentially located viscoelastic elements, or a meshwork of viscoelastic elements throughout the CCS. A similar approach was used by Odell et al (1981) for a different cuboidal epithelium.

As outlined in the above, experiments have identified that the CCS of the columnar epithelium has a ring of force producing contractile fibres surrounding the cir- cumference of each cell. These fibres could be considered to drive the majority of movement of the columnar epithelium CCS. However, a variety of other potential mechanisms have been identified, which may also play a prominent role. These include other experimentally identified sub cellular structures that produce forces and movement in and around the CCS, and theoretical geometric considerations such as conservation of cell volume or area. This leads to the questions: what impact do circumferential contractile fibres have on columnar epithelium CCS movement, and can they produce the majority of the CCS movement?

The epithelium models provide a representation of the movement of contractile fibres at the tissue scale. There is, however, other work that investigates the movement of contractile fibres as an individual system. This work provides insight into both the stress strain relationship (Deguchi et al, 2006; Matsui et al, 2009, 2013) and contractile properties (Hill, 1938; Besser and Schwarz, 2007; Stachowiak and O'Shaughnessy, 2008; Colombelli et al, 2009; Stachowiak and O'Shaughnessy, 2009; Kaunas et al, 2011; Thoresen et al, 2011) of individual contractile fibres that are not captured in current epithelium scale models. This raises the questions: can a description of epithelial scale contractile fibre forces and movement be developed based on research focused on contractile fibres as an individual system, and can such a description provide further understanding of columnar epithelium mechanics?

In this work, we investigated the questions outlined above by developing an alternate vertex description of columnar epithelium CCS mechanics, where the cross section movement is driven from the forces and movements of circumferential contractile fibres without any other potential mechanisms. Our description of the circumferential contractile fibre mechanisms was developed from work focussed on contractile fibres as an individual system. Additionally, in our model, circumferential contraction occurred from a series of contractile fibres connected between vertex points, rather than one unit extending around the periphery of each cell.

We tested the generality of our model by seeing if it predicts two different laser ablation datasets (taken from Farhadifar et al (2007) and Landsberg et al (2009)) with the same parameters. We exactly matched our simulations' epithelium geometry to those in the experiments, testing whether the model can predict the exact response of each individual dataset rather than a distribution of responses. The match of simulation to experiment also allowed us to more accurately investigate the relative impact of different mechanisms of movement. Once we tested the model, we explored its predictions of mechanisms which have a substantial 
impact on columnar epithelium movement. This included cell conservation of area, the impact of global sheet movement, and an unknown mechanism that could be related to minimum cell area or contractile fibre lengths. We also further demonstrated the usefulness of our model by investigating the relationship between contractile fibre strain (or tension) and movement after laser ablation.

\section{Experimental Data Analysis Methods}

The experimental datasets used in this paper are taken from Farhadifar et al (2007) Supplemental Movie S4 and Landsberg et al (2009) Supplemental Movie S2. They are of the laser ablation of columnar epithelial cells located in the third instar larval wing disc of Drosophila, and are referred to as the primary and validation datasets respectively. Details of image analysis procedures used are described in the Online Resource Methods.

\section{The Model}

A schematic of a columnar epithelium is shown in Fig. 1a. The CCS is located in the top region of the cell (apically), and is shown in more detail in Fig. 1b. Considerable research (Ebrahim et al, 2013; Owaribe et al, 1981; Langanger et al, 1984; Drenckhahn and Dermietzel, 1988; Sandig and Kalnins, 1988; Yonemura et al, 1995; Smutny et al, 2010) has shown that circumferential contractile fibres are located directly adjacent to cell membranes.

\subsection{Epithelium Scale Model}

We defined a two dimensional epithelium scale model focused on the forces created by circumferential fibres. It is well accepted that large viscous forces occur in systems at the length scale of epithelial cells and tissues (Odell et al, 1981; Fletcher et al, 2014), and therefore they were also included. In our model, these viscous forces can be considered to arise from the resistance of the cytoplasm, and its various cell structures, to movement created by the circumferential contractile fibres. The mechanisms of our model are different to other columnar epithelium models, in that we do not include any geometry related terms, such as conservation of volume or cell area elasticity.

As depicted in Fig. 1b, we used a vertex representation of the system (Weliky and Oster, 1990). This representation recognises the relatively polygonal shape of an epithelium, where the peripheries of cells are a series of relatively straight segments connected together by points where three or more cells meet. Herein, the straight segments and points are called cell edges and vertices respectively.

Circumferential contractile fibres produce forces tangent to their length and are situated along cell edges. Therefore we treated the contractile segment along each cell edge as an individual fibre which applied forces to the vertices located at each end. This is similar to the way circumferential contractile fibre forces are applied in Weliky and Oster (1990) and Weliky et al (1991), though is different to more recent columnar epithelium models where the circumferential contractile fibres are treated as one unit via periphery length terms. This approach approximates that all contractile fibres in the epithelium show the same forces and movement regardless of their length. This approximation is supported by experimental evidence that contractile fibres of different lengths have the same properties for their force versus contraction relationship (Thoresen et al, 2011), stall force (Thoresen et al, 2011), response to tension (Deguchi et al, 2005; Matsui et al, 2009; Kaunas et al, 2011; Matsui et al, 2013) and total length reduction after contraction (Katoh et al, 1998; Colombelli et al, 2009). It is noted that some experimental evidence suggests that the maximum retraction velocity of contractile fibres under no tension can vary between fibres of different lengths (Thoresen et al, 2011).

Based on the above assumptions, we described the movement of the epithelium from equations of motions at each vertex as:

$\mu \overrightarrow{\dot{x}}=\sum_{d=1}^{D} \vec{F}_{d}^{C F}$

where $\vec{x}$ is a two dimensional vector representing the location of the vertex, $\vec{F}_{d}^{C F}$ are the contractile fibre forces, $\mu$ is a damping constant, and the subscript $d$ represents the edges associated with the vertex. Here we also applied the common overdamped assumption (Fletcher et al, 2014), where inertial forces of an epithelium are considered to be negligible relative to viscous forces.

\subsection{Contractile Fibre Scale Mechanisms}

The epithelium scale model provides a framework in which contractile fibre mechanisms drive epithelium movement. There is a large body of experimental and theoretical literature which has investigated the movement of contractile fibres as an individual system. This work 

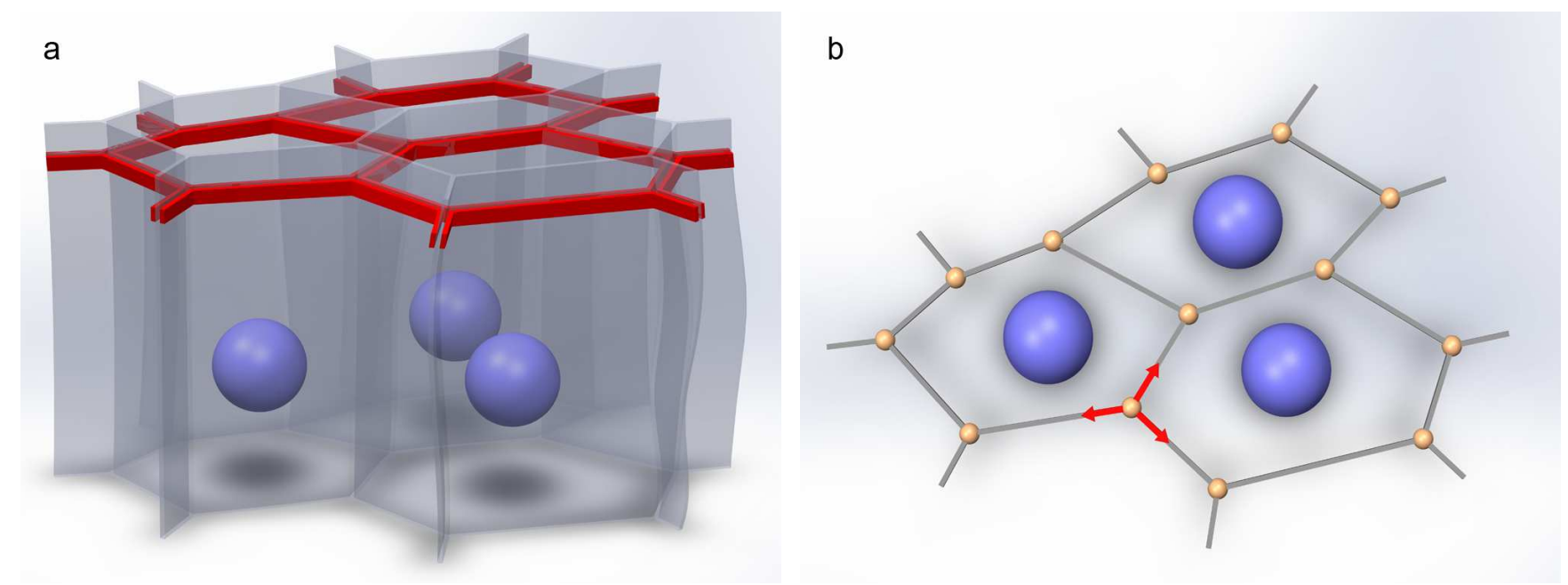

Fig. 1: a Schematic of simple columnar epithelium showing cell membranes (grey), cell nuclei (blue), and CCS circumferential contractile fibres (red). b Two dimensional view of CCS also showing vertices (yellow), and forces from cell membrane adjacent contractile fibres (red).

provided insight into the mechanisms controlling the movement of contractile fibres. It is focussed on contractile fibres from various non-muscle cells. The contractile fibres that occur in non-muscle cells are highly similar to those that occur in the CCS of the columnar epithelium. They are made up of the same proteins (Wehland and Weber, 1980; Geiger et al, 1981; Sanger et al, 1983; Drenckhahn and Dermietzel, 1988), show the same sarcomeric type pattern (Sanger et al, 1983; Langanger et al, 1984), and contract in the same manner (Owaribe et al, 1981; Katoh et al, 1998; Kumar et al, 2006). The only difference is that fibres of general non-muscle cells have an additional cross linker called Filamin (Wang et al, 1975; Wu et al, 2012). As such, we based our epithelial circumferential contractile fibre description on the non-muscle contractile fibre literature.In this literature, contractile fibres show both tension and contraction mechanisms of movement, which we therefore include in our contractile fibre model.

\subsubsection{Tension Mechanism}

The tension mechanism of our contractile fibres was developed based on the experimental work of Deguchi et al (2005), Matsui et al (2009) and Matsui et al (2013). They applied increasing tension to isolated contractile fibres in the absence of myosin mediated length reduction. They found that the fibres had an approximately linear stress strain relationship, and could undergo strains of up to three without breaking. We note that some experimental evidence suggests that contractile fibres may show some strain stiffening behaviour at high strains (Deguchi et al, 2006). Based on this research, we assumed linear elastic behaviour of contractile fibres as shown in Eq. 2. We further assumed the same Cauchy definition of strain as used in those experiments. In this strain definition, structures are represented as lines with a tension proportional to the ratio of the extension of a contractile fibre over its unstrained length. This means that shorter fibres will produce greater tension than longer ones, when stretched the same amount. Contractile fibres have also been treated as a linear elastic material with a Cauchy definition of strain in individual contractile fibre literature (Kaunas et al, 2011), where they investigated fibre behaviour under cyclic strain. Most other columnar epithelium models differ from our contractile fibre tension representation by defining line tension as either constant along cell edges (Hutson et al, 2009) or proportional to an extension of the cell edges from a resting length (Solon et al, 2009; Jayasinghe et al, 2013) in force descriptions, or as directly relative to the length of cell edges (Farhadifar et al, 2007) in energy descriptions.

As in other epithelium scale models, our tension mechanism included any tension resistance created by other cell structures. Any such resistance would be from circumferentially located actin filaments, or the spectrin cytoskeleton which supports the cell membrane (Drenckhahn and Dermietzel, 1988). It is reasonable to assume that these structures can be represented with the Cauchy definition of strain, especially as actin has been experimentally found to have a similar elastic tension response as contractile fibres (Kojima et al, 1994). The closest epithelium scale tension description to ours 


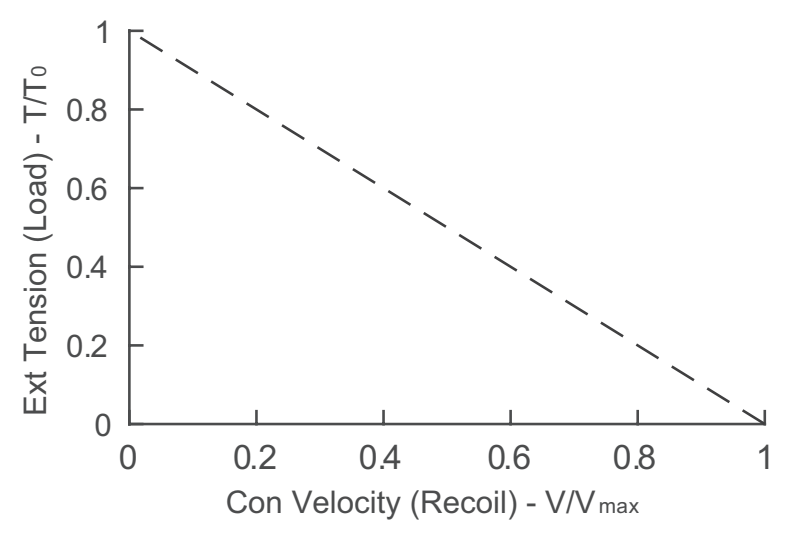

Fig. 2: Model relationship between contractile fibre external tension (load) and contraction velocity (recoil). $T_{o}$ represents the maximum tension at which contraction will occur. $V_{\max }$ represents the maximum contraction velocity.

is from Hufnagel et al (2007), who used the ratio of relative extension squared to the preferred perimeter length for cell perimeter extension.

As contractile fibres reduce their length through myosin mediated contraction, we considered the strain as relative to a contractile fibre minimum length, rather than the initial length used in the standard definition of Cauchy strain. We defined the minimum length as the shortest length that a contractile fibre has achieved up to the current simulation time, and as such it is a dynamically changing variable. Similar fibre minimum length assumptions have also been applied to individual contractile fibres by Kaunas et al (2011).

\subsubsection{Contraction Mechanism}

In individual non-muscle contractile fibre work, it is common to base non-muscle contractile fibre mechanisms on research conducted into muscle fibres in the 1930 s and 1940s. For the contractile mechanism of our fibres, we utilised a linear approximation of the recoil versus load relationship originally found for muscle in Hill (1938). Recent experimental investigations have also found this linear recoil versus load relationship for non-muscle contractile fibres (Thoresen et al, 2011). In the load versus recoil relationship, the rate of contraction depends on the external load applied to the fibre, as shown in Fig. 2. At zero load, the fibre contracts at a maximum rate. The contraction rate then decreases with increasing load until a stall force is reached, at which the fibre no longer contracts. In our model (Eq. 2 ), the external load on an individual contractile fibre was represented by the forces that other fibres apply to the vertex point (Fig. 2).
Our model of contractile fibres assumed that myosin mediated contractile forces were applied directly to vertices, when physically such forces would be applied within the fibre. While this meant that contractile forces would actually be applied to vertices through a tension rather than contraction force, it was not expected to make any difference for the epithelium scale movement of the model.

In other columnar epithelium models, contractile behaviour is commonly included with general line tension curves proportional to cell edge length or perimeter contractility relative to cell perimeter extension, rather than the load recoil relationship we have used. It is noted that the Solon et al (2009) and Jayasinghe et al (2013) models of flat amnioserosa also treat circumferential contractile fibres as a series of individual units. However their description of these fibres are different, in that they: use a spring representation rather than a general strain definition; have the same nondynamic minimum length for all springs; and represent spring contraction forces via a mathematical Hill equation (Goutelle et al, 2008) rather than the load recoil representation we have used.

The contractile fibre and tension behaviours described were combined with the epithelium scale model via:

$\overrightarrow{\dot{x}}_{v}=\sum_{d=1}^{D}\left(\frac{\vec{F}_{d}^{C o n}}{\mu}+\frac{A E}{\mu} \frac{\left\|\vec{L}_{d}\right\|-L_{m i n, d}}{L_{m i n, d}} \frac{\vec{L}_{d}}{\left\|\vec{L}_{d}\right\|}\right)$

where $v$ is the vertex number, and for each edge, $\vec{F}_{d}^{C o n}$ is the contraction force, $A$ is the representative cross sectional area, $L_{m i n, d}$ the minimum length, $E$ is the elastic modulus, and $\vec{L}_{d}$ is a vector representing the length and direction of the edge $\left(\vec{L}_{d}=\vec{x}_{d}-\vec{x}_{v}\right)$. Note $\vec{L}_{d} /\left\|\vec{L}_{d}\right\|$ determines the directionality of the elastic force vector.

\subsection{Parameters and Solver}

We assumed that each edge has the same elasticity cross section, area and contraction force, which results in two model parameters. These parameters represent the ratio of contractive forces to damping (referred to herein as the contraction force parameter) and the ratio of elastic forces to damping. The equations of motion were solved in Matlab via custom written code using the Explicit Euler Method (Butcher, 1987). This method was found to be stable and accurate for all simulation runs. 


\subsection{Matching Simulation Geometry to Experiments} via Initial Conditions

For each simulation, we matched the model geometry to that of the experiment just prior to laser ablation. As an initial condition, the simulation vertices were assumed to have the same location as the corresponding experimental vertices. The model was then allowed to reach steady state, after which laser ablation was induced (by removal of the edge corresponding to the experimentally severed one). Initially we attempted to match the simulation pre-ablation steady state to the corresponding experimental geometry assuming uniform minimum length $\left(L_{\text {min }}\right)$ initial condition for all edges in the sheet. However it was not possible to achieve a close match with this approach (refer to Online Materials).

We instead used a variation of force-inference methods (Brodland et al, 2014; Ishihara et al, 2013). Forceinference techniques use optimisation approaches to infer cellular forces from cell shapes. In the optimisation used here, we used the $L_{\min }$ values for each edge as fitting parameters, and a vector of the magnitude difference between model and experimental vertex location$\mathrm{s}$ as the cost function. For this optimisation, we used an inbuilt Matlab non-linear least squares constrained approach (lsqnonlin). Our approach varies from other force-inference work, as we solve for the $L_{\min }$ parameters controlling edge strain, rather than forces directly.

Our force-inference approach achieved a very close match between model and experiment, which is shown in the Online Resources. As in other force inference work (Brodland et al, 2014), it predicted a highly variable strain distribution (refer to sheet $L_{\min }$ and strain distribution in the Online Resources). This highly variable sheet strain distribution is commonly assumed to arise from a combination of edge lengthening and shortening mechanisms (Brodland et al, 2014; Brodland, 2004; Lecuit et al, 2011). Specific to our model, we assume that the strain distribution arises as a result of the nonuniform geometry of the sheet edge, combined with the cell proliferation process that produces the epithelium. Whenever a cell proliferates, it creates a new edge. This edge reduces in length until it is in force equilibrium with the surrounding geometry. As the surrounding geometry, and the forces it applies to a specific edge, vary throughout the sheet, so will the strain and $L_{\min }$ values required to be in equilibrium.

\subsection{Boundary Conditions and Global Sheet Movement}

Vertices near the edge of the experimental microscopy images were treated as boundary points. Cell edges

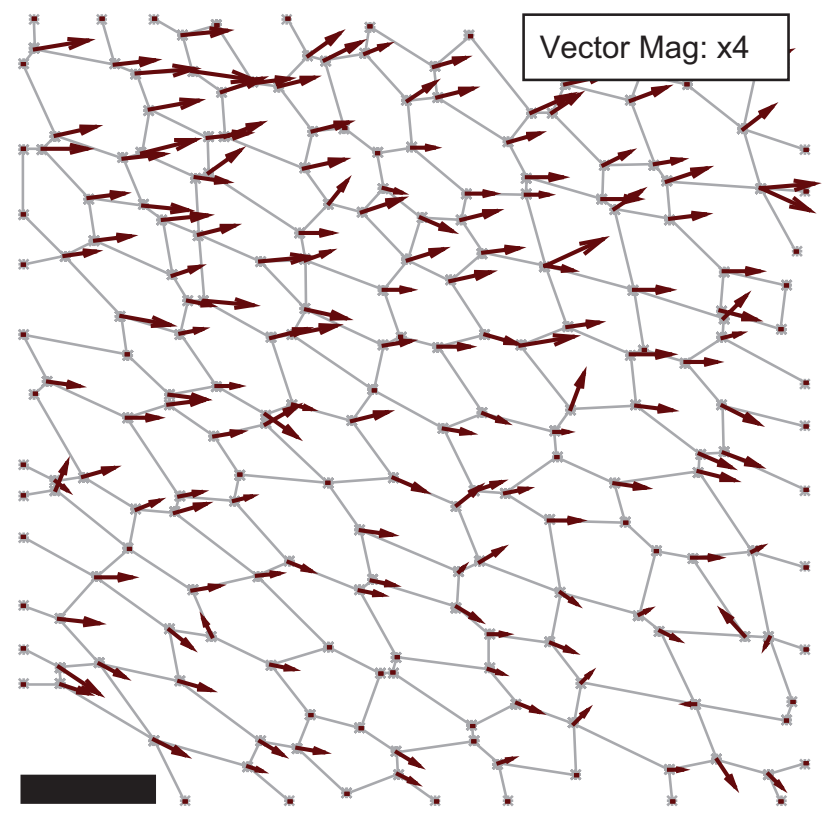

Fig. 3: Global sheet movement of primary dataset occurring prior to laser ablation (0 to $240 \mathrm{~s}$ ). Displacement vectors represent four times actual displacement. Scale Bar: 5 microns.

which intersect the perimeter of microscope images were not considered to be boundary points. Throughout the course of the experiment, all vertices showed considerable movement not related to laser ablation. This movement was most obvious in the experimental images prior to laser ablation (Fig. 3). Here vertices throughout the sheet were moving in a non-uniform manner. As the sheet has not yet been ablated, this movement cannot be related to laser ablation, and therefore must be a general movement of the sheet. It has been shown in other work (Mao et al, 2013) that larval Drosophila wing discs have global patterns of tension, which would cause the large scale movements seen in experiments. Herein, this movement is referred to as global sheet movement, which is defined as experimental sheet movement not related to laser ablation of a cell edge. We incorporated global sheet movement into the model by translating the simulation boundary points in the same way as their experimental counterparts.

\section{Results}

The primary dataset response to laser ablation of the CCS of a simple columnar epithelial cell system is shown in Fig. 4. This is a typical epithelial sheet response. The laser is aimed at the edge of two cells between vertices, and severs the contractile fibres of both cells on that 


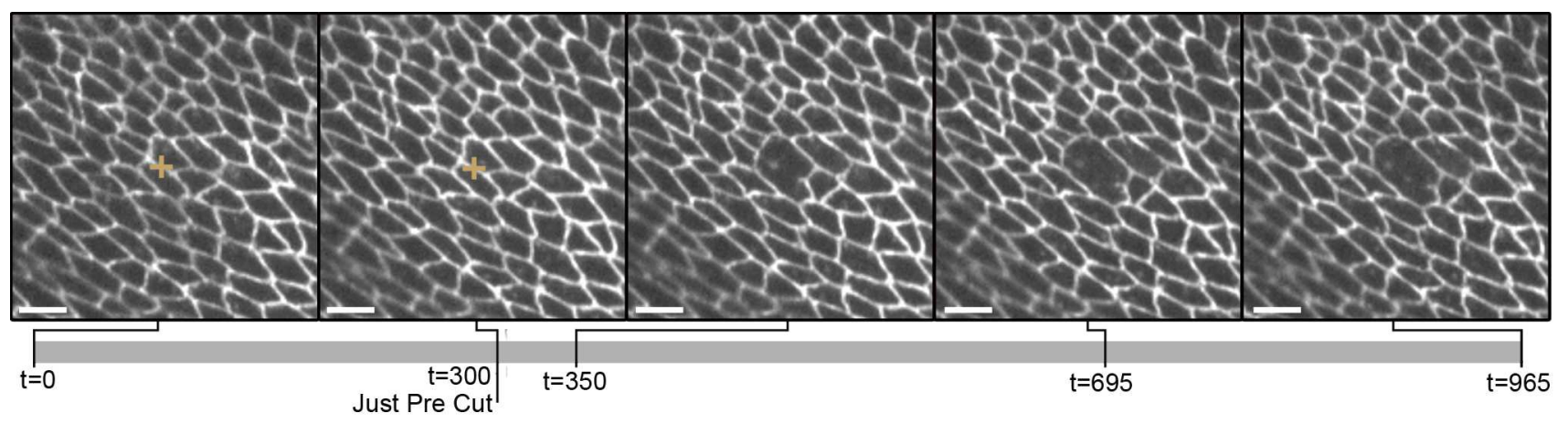

Fig. 4: Experimental microscopy images of CCS of columnar epithelial cell sheet from primary dataset at different time points. Scale Bar: 5 microns. Brown cross represents laser cut location.

edge. After ablation, the sheet moves or relaxes away from the cut point.

\subsection{Model Testing}

To test the model, we first investigated whether the model can predict the primary dataset. For this, the two parameters of the model were matched to the primary dataset output $\left(F^{C o n} / \mu=2.36\right.$ and $\left.E A / \mu=3.94\right)$; predominantly using the average root means squared displacement, though also the severed edge movement.

Fig. 5 shows a comparison between the model prediction and experimental movement of the primary dataset. In Fig. 5a and Fig. 5b, it can be seen that the model reproduced the experimental trends of anisotropic movement of the experimental sheet (where vertices move more in the parallel compared to orthogonal direction of the severed edge), and reducing movement of vertices at further distances for the severed edge. Additionally, the model reproduced the distance that laser ablation induced movement spreads from the cut point (four cells).

The model prediction of individual vertex movement over the laser ablation response was quantitatively compared to experiment in Fig. 5c. Here the vertices were grouped based on their distance from the cut point (as shown in Online Resources). The radial and angular displacement of each vertex was plotted against its angular location relative to the ablated join. As can be seen, the model quantitatively reproduced individual vertex movement over the laser ablation response.

The amount of movement of the model over time was compared to experiment via the average root mean squared displacement and the severed edge length change, as shown in Fig. 5d. Here the model reproduced the amount of movement of the experimental sheet within experimental error throughout time. The model also reproduced the dynamics of the experimental response within experimental error, and importantly captured the fast initial movement of the sheet (300 to 350 seconds).

A comparison of severed edge length change between the model with global sheet movement and experiment is shown in Fig. 5e. Here the model reproduced the general trend of length change of the severed edge over time; predicting a fast initial response, followed by a slowed response. The model also predicted the final length changes of the experiment within experimental error, though over predicted the magnitude of the initial response. This can also be seen in Fig. 5b, where the model severed edge vertex on the lower right moved considerably more than the experimental one.

To complete testing, and check for generality, the model was used to predict the movement of the validation dataset with the same parameters as the primary dataset. The validation experimental dataset was slightly different to the primary dataset. It showed less of the sheet and did not include the final steady state of the sheet after the laser ablation response. The details of the validation dataset model to experiment comparison are in the Online Resources Results. Aside from the sheet final steady state post laser ablation, which could not be compared, the model reproduced all the same trends and quantitative matches for the validation dataset as seen in the primary dataset using the same parameters.

\subsection{Contractile Fibre Forces During Laser Ablation}

The forces of two representative edges during the initial laser ablation response are shown in Fig. 6. These forces demonstrate that edge tensions change significantly during the laser ablation response. During the initial laser ablation response, twenty three edges not located near the boundary showed greater than five 

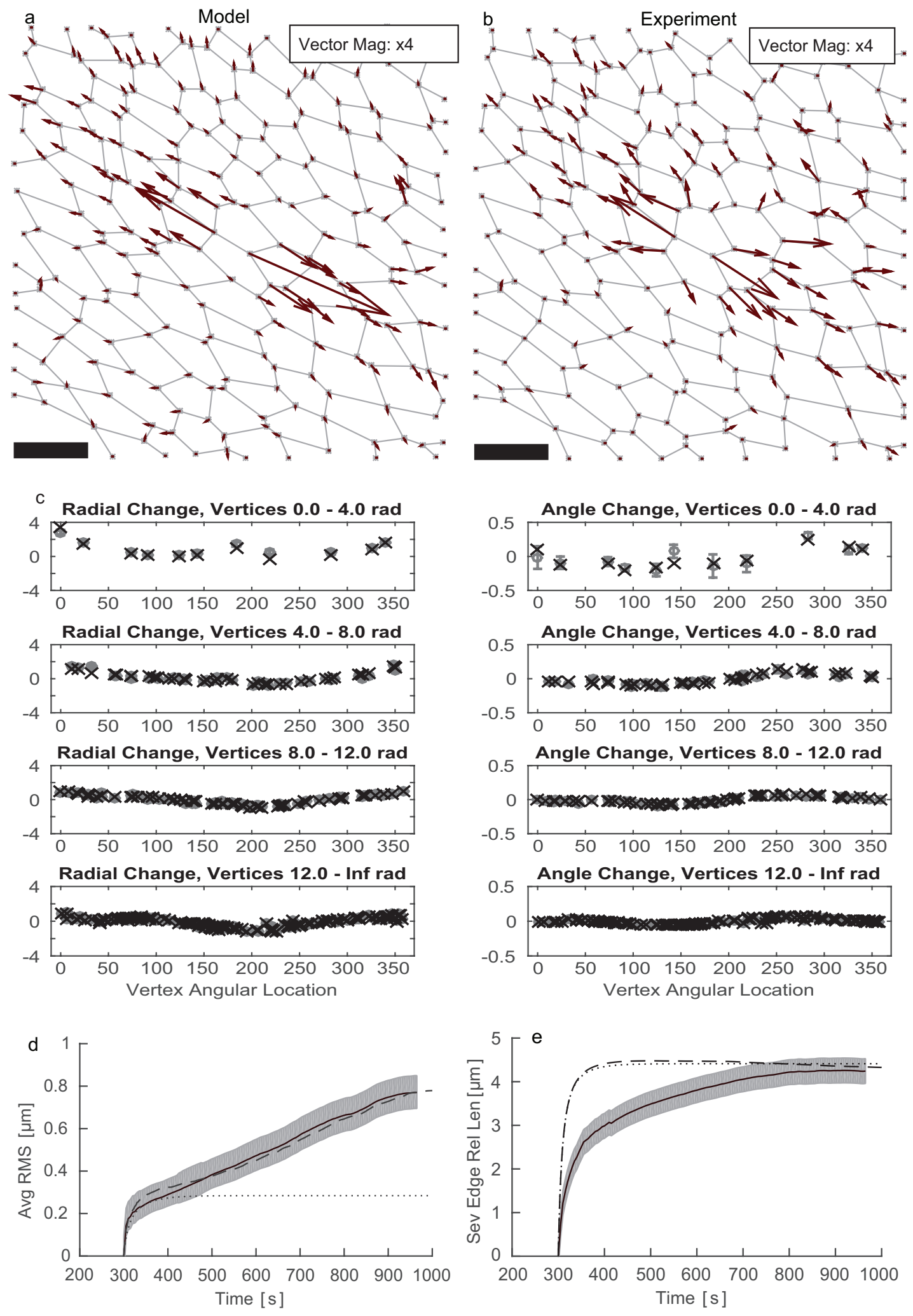

Fig. 5: a \& b Comparison of model prediction a to experiment b for primary dataset vertex movement from just prior to laser ablation (300 s) to $350 \mathrm{~s}$. Displacement vectors represent four times actual displacement. Scale Bar: 5 microns. c Quantitative comparison of the radial and angular movement of vertex points from just prior to laser ablation to the completion of the laser ablation response of the primary dataset. Black crosses represent model predictions. Grey circles represent experimental movement. Horizontal axis represents vertex angular location relation to the cut join, and vertices are grouped based on distance from the cut point (refer to Online Resources). d \& e Quantified comparison between model with global sheet movement (dashed line), model without global sheet movement (dotted line) and experiment (solid line) for primary dataset movement after laser ablation. $\mathbf{d}$ Average Root Mean Squared displacement of all vertices from just prior to laser ablation over time. e Severed edge length relative to length from just prior to laser ablation over time. Shaded region represents experimental error. Data is smoothed via a windowed moving average. 
percent tension change. Fig. $6 \mathrm{~b}$ and Fig. 6c also show edges undergoing contraction mediated length reduction. When the external load (grey line) is below the contraction forces (dashed line) the relative external tension ratio $\left(T / T_{o}\right)$ is less than one and the edge is reducing in length via contraction. During the laser ablation response eleven edges not located near the boundary had contraction mediated length reduction, with eight edges recoiling at faster than five percent of the maximum contraction velocity. Many of these edges were located adjacent to the severed edge (four of the five edges connected to the severed edge), and six of them had resultant minimum length reductions.

\subsection{Epithelium Mechanisms of Movement During} Laser Ablation

After testing of the model, we used it to investigate three potential columnar epithelium mechanisms of movement: cell area change, global sheet movement, and a kink which was not predicted by the model but occurred in experiments.

The cell areas predicted by the model were investigated by calculating the area of each cell over the course of the simulation. Many of the cells were found to change area by greater than 5 percent. Two cells especially, cells 4 and 5 in Fig. 7, showed approximately 20 percent area change over the simulation of laser edge severing. This finding was verified experimentally using the primary dataset. It was found that nine cells had statistically significant cell area changes throughout the course of the experiment (Fig. 7). The largest area change occurred in cell 5 , which had a $60 \pm 14$ percent reduction in area. The area of cell 5 over time is shown in the Online Resources.

To investigate its impact on the system, global sheet movement was removed from the model. The simulated response of the primary dataset to laser ablation without global sheet movement is shown by the dotted line in Fig. 5d and Fig. 5e. In Fig. 5d, it can be seen that the average root mean squared movement of the sheet flattened out at approximately 400 seconds (100 seconds after laser ablation), and did not continue the upwards trend of the experimental data. This indicated that the experimental response was dominated by global sheet movement after 400 seconds, which was also verified in the experimental data. Fig. 8 shows the impact of global sheet movement on the experimental response. As shown in Fig. 8a, initially the laser ablation response caused sheet relaxation towards the top left of the sheet. However, over the course of the experimen$\mathrm{t}$ global sheet movement caused the vertices to move back to the right (Fig. 8b), and completely overrode the laser ablation response. As shown in Fig. 5e, the

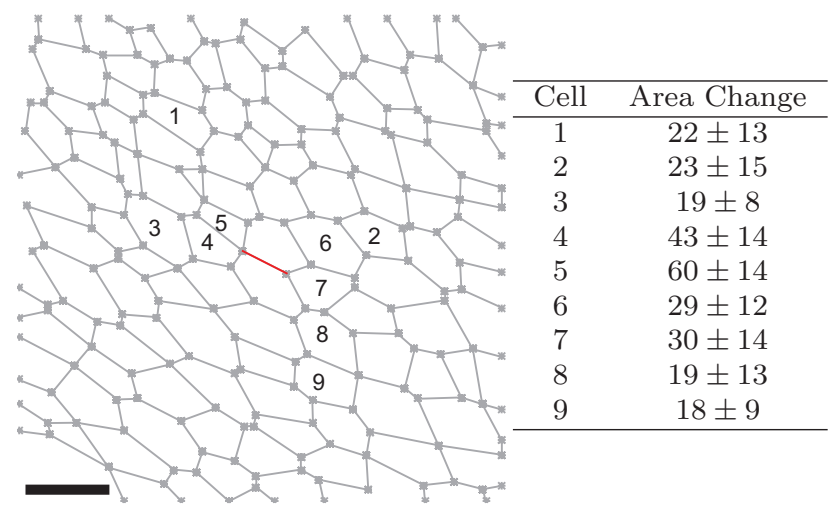

Fig. 7: Cell area changes of primary dataset during experiment. Cells with statistically significant error change identified on left. Severed edge shown in red. Scale Bar: 5 microns. Maximum area change for significant cells on right. Error is two standard deviations of a Gaussian distribution.

global sheet movement appeared to have only a minor effect on the length change of the severed edge. Similar global sheet movement was identified in the validation dataset; refer to Online Resources.

The simulations also showed that the model did not reproduce a kink that occurs in the experimental data. This can be seen in Fig. 9, where the experimental cell edges near the laser ablated edge have a kink (b), but the model prediction does not (c).

\subsection{Contractile Fibre Strain/Tension and Laser Ablation Sheet Movement}

We used our model to investigate the relationship between the strain of an individual edge and its movement upon laser ablation. This was achieved by simulating laser ablation of various centrally located edges in the primary dataset. We found a strong correlation between the stain of an edge and its movement when severed (Fig. 10). As shown, higher strain lead to larger movement, and this relationship appeared to be linear.

\section{Discussion}

In this work, we found that our model reproduced all the trends of sheet movement, and quantitatively predicted both the final sheet movement and the total sheet movement throughout the laser ablation response. This indicates that the majority of CCS movement of the columnar epithelium can be driven by circumferential fibres, without any other potential columnar epithelium mechanisms of movement. 

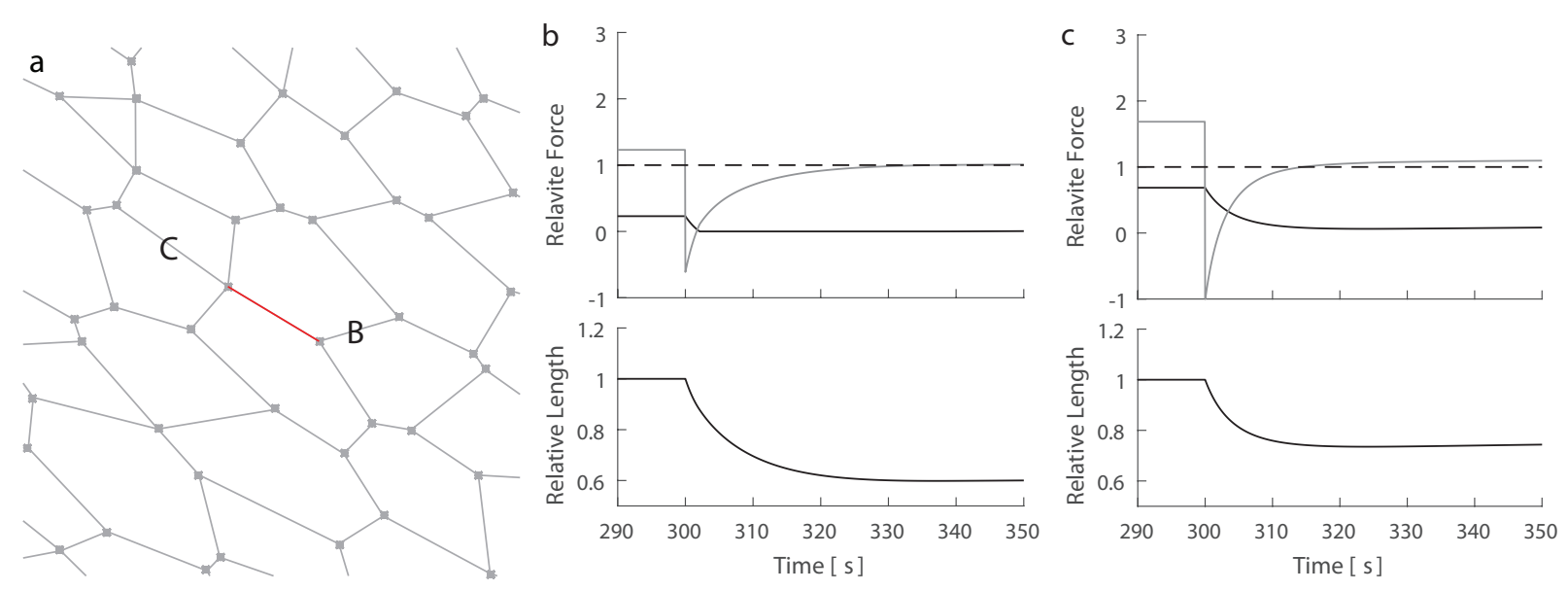

Fig. 6: Relative forces and length change of representative edges during the fast initial movement following laser ablation (from just prior to laser ablation to 350 seconds). a Location of representative edges relative to the severed edge highlighted in red. b \& c Relative forces and length change of edges shown as $\mathbf{B}$ and $\mathbf{C}$ in $\mathbf{a}$ respectively. For relative forces, solid black lines, dotted lines, and solid gray lines represent tension forces, contraction forces, and external loads on each edge respectively. Relative force and length are scaled by the contraction force parameter and edge length prior to laser ablation respectively.
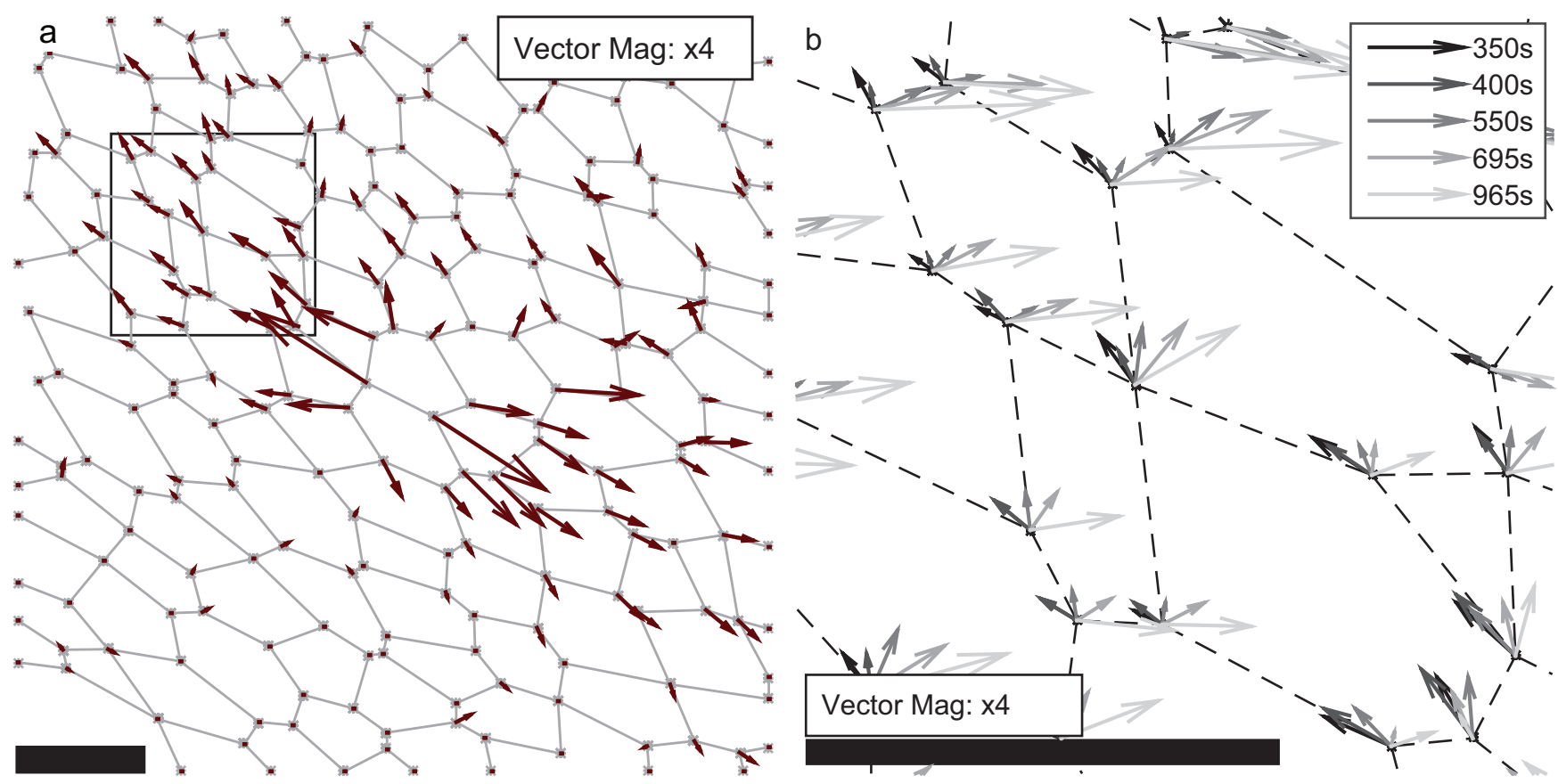

Fig. 8: Impact of background movement on experimental vertices in primary dataset post laser ablation. a Whole sheet view from just prior to laser ablation (300 s) to $350 \mathrm{~s}$. b Magnified view of box shown in A. Movement from $300 \mathrm{~s}$ to times in legend. Displacement vectors represent four times actual displacement. Scale Bar: 5 microns. 


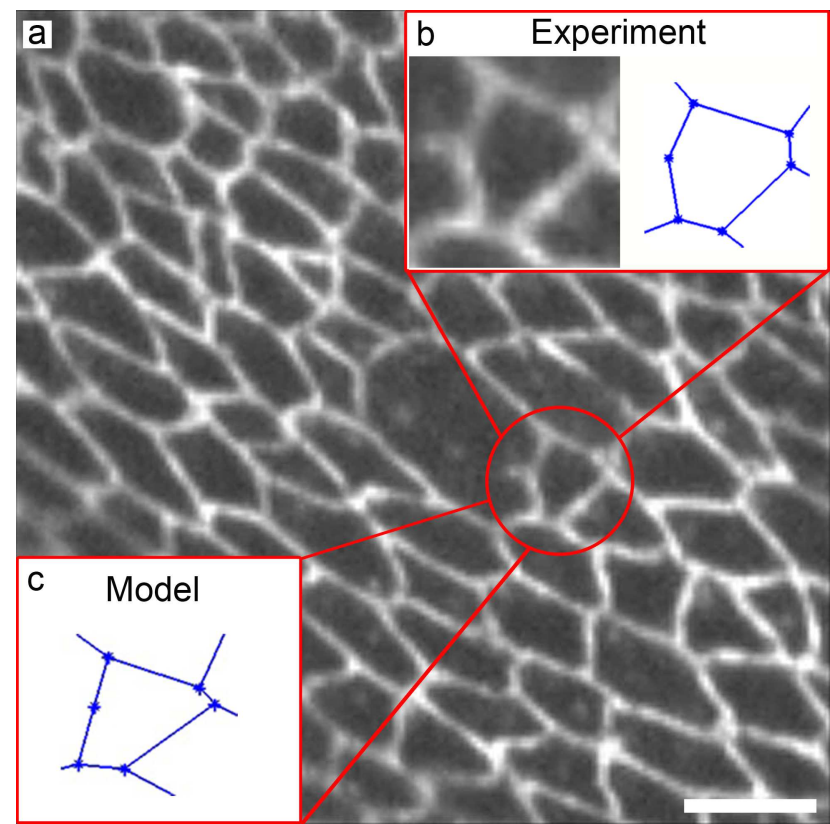

Fig. 9: Non-straight kinked stopping of cell edges near severed edge in primary dataset at $965 \mathrm{~s}$. a Whole sheet microscopy view. b Magnified view of kinked cell edge from microscopy image (left) and experiment image analysis (right). c Magnified view of model prediction at kinked cell edge region of experimental data. Scale Bar: 5 microns.

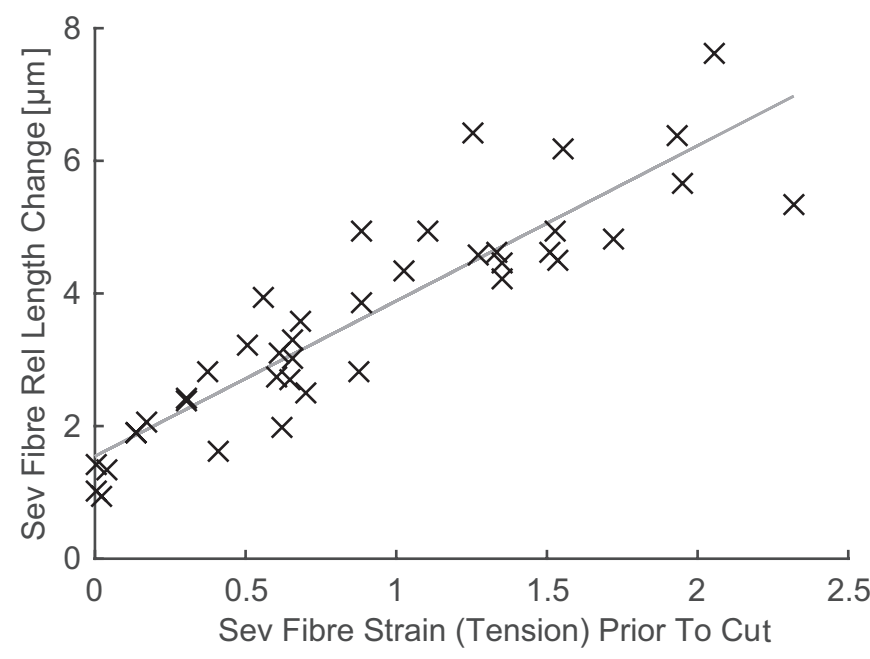

Fig. 10: Plot of relationship between individual fibre strain (or tension) prior to laser ablation and final fibre relative length change after severing. The grey line represents a best fit linear approximation.
The model was less accurate in the immediate vicinity of the laser ablation cut, predicting that sheet movement in this region would occur much faster than in experiments. It is likely that this inaccuracy results from modeling assumptions related to the elastic recoil of contractile fibres. Some research into the movement of individual contractile fibres indicates that when a fibre is relaxing from a strained position, it does so in a time dependent manner (Stachowiak and O'Shaughnessy, 2009). Our model did not capture this. It assumed that any elastic recoil is immediate. The introduction of time dependent elastic recoil should significantly slow down the models prediction of sheet response in the immediate vicinity of the last ablation cut points, causing the model to more accurately match experiments.

Our modeling approach is based on matching the simulation to experimental geometry, allowing exact comparison of model prediction to experimental movement. Other columnar epithelium models are not matched to experimental geometry, and therefore are only compared via distributions of responses. This makes it difficult to compare model results. The most appropriate comparison can be drawn with (Farhadifar et al, 2007) who also evaluated their model's predictions based on final radial and angle vertex displacements (as in Fig. 5) of the primary dataset. The (Farhadifar et al, 2007) model reproduces many of the experimental laser ablation response trends, though in the radial displacements the model appears to over-accentuate the anisotropy of the experimental response, especially at distances far from the severing location. Also the (Farhadifar et al, 2007) model angular displacements appear to be larger than experiments close to the severing location, but smaller than experiments far from the severing location. In comparison, our model reproduces all trends of the final sheet movement.

The success of our model in predicting the movement of the columnar epithelium CCS indicates that an epithelium scale contractile fibre description can be developed based on research focused on contractile fibres as an individual system. Our model also predicts that during laser ablation cell edges can have both significant tension changes and undergo contraction mediated shortening. This shows that our contractile fibre based modeling assumptions affect epithelium scale movemen$\mathrm{t}$ of the model during the laser ablation response. We also matched our simulation to experimental geometry. This provided a rigorous test of whether the model could predict the laser ablation response, and allowed us to evaluate the exact impact of various epithelium mechanisms on sheet movement. We initially found that this matching could not be achieved using a uniform minimum length for all edges, and therefore we devel- 
oped an alternate version of force inference methods to achieve a close match.

The epithelium mechanisms of movement that we evaluated were: columnar epithelial cell conservation of area, global sheet movement, and an unexpected mechanism that resists cell edge movement in certain situations.

Cell conservation of area is a common geometric assumption that has been used to model the movement of the CCS of columnar epithelial cell during laser ablation. Conservation of area has also been experimentally investigated in (Hutson et al, 2009) for flat amnioserosa cells. In that work, they found that cells near the laser ablation location conserve area on short time scales. The majority of those cells showed up to six percent change in area, with the two cell on either end of the severed edge showing up to ten percent area change. Our model predicted that columnar epithelial cells do not conserve area during the laser ablation response. We validated this model prediction using the primary dataset, finding cell area changes of up to sixty percent. This experimental data shows that columnar epithelial cells do not conserve area on the time scale of the laser ablation response. It also demonstrates a further difference between the movement of columnar and flat epithelial cells.

It is well known that global sheet movement occurs in general epithelial tissue undergoing shape changes (morphological events) (Butler et al, 2009). This movement has also been recognised in the Drosophila Wing Disc columnar epithelium (Farhadifar et al, 2007) while it maintains a steady shape. In the wing disc, global sheet movement is thought to occur as a result of global tension patterns resulting from uneven cell proliferation throughout the disc (Mao et al, 2013). During development of our model, we found that global sheet movement causes non-homogenous movement in small areas of the sheet. Our model also predicted that during laser ablation global sheet movement can dominate the movement of the epithelial sheet close to the severed edge. These findings have quantified the impact of global sheet movement on the epithelium, and provided further evidence that global sheet movement is a significant mechanism that impacts sheet movement on the time and length scales of laser ablation. Additional, these finding suggest that if global sheet movement is to be taken into account, non homogenous methods such as moving boundary points should be used.

When simulating the primary dataset, the model predicted an additional movement mechanism that resists cell movement. This mechanism was identified near the laser ablated edge, where the experimental results stopped in a kinked position, which the model did not reproduce. This indicates that there is some unknown mechanism in the experiment that is not included in the model. This kink occurred where both contractile fibres and cell areas had reduced by a large amount. Therefore the mechanism could be related to a minimum length of contractile fibres, which has been experimentally reported in individual contractile fibre literature (Katoh et al, 1998; Deguchi et al, 2005; Thoresen et al, 2011), or some kind of internal cell resistance at a minimum area. Resistance of cells to large sheet movements was also found experimentally in Owaribe et al (1981) where they stimulated extreme sheet contraction, though has not been reported for physiological or laser ablation induced movement of a columnar epithelium. Our work shows that this cell resistance mechanism occurs during laser ablation, and may be required to completely capture general columnar epithelium CCS movement.

As our model provided individual strains, and accurately predicted final sheet movement, we used it to investigate the relationship between edge strain and final edge movement after laser ablation. In experimental approaches, it is common to use the movement of an individual edge after laser ablation as an estimate of the tension which that edge is under. When doing this, the tension (or strain) of an edge is calculated based on its initial recoil velocity after ablation. Our model predicted a strong linear relationship between them. This finding indicates that, based on simulations, the final movement of an edge can also be used to estimate individual edge pre ablation strain. The recording of edge final movement could also be less reliant on measurement technique than initial recoil velocity, and therefore would be a more robust measure of pre ablation edge strain.

Acknowledgments This research was supported by the Victorian Life Sciences Computation Initiative (VLSCI) grant UOM0012 on its Peak Computing Facility at the University of Melbourne, an initiative of the Victorian Government. We would also like to acknowledge the statistical support of the Statistical Consulting Centre at the University of Melbourne, specifically Rachel Sore.

\section{Compliance with Ethical Standards:}

Funding: This study was funded by the Australian Government Department of Education and Training Australian Postgraduate Award.

Conflict of Interest: The authors declare that they have no conflict of interest.

\section{References}

Aegerter-Wilmsen T, Smith AC, Christen AJ, Aegerter CM, Hafen E, Basler K (2010) Exploring the effects 
of mechanical feedback on epithelial topology. Development 137(3):499-506

Aliee M, Rper JC, Landsberg KP, Pentzold C, Widmann TJ, Jlicher F, Dahmann C (2012) Physical mechanisms shaping the drosophila dorsoventral compartment boundary. Current biology 22(11):967976

Bambardekar K, Clment R, Blanc O, Chards C, Lenne PF (2015) Direct laser manipulation reveals the mechanics of cell contacts in vivo. Proceedings of the National Academy of Sciences p 201418732

Besser A, Schwarz US (2007) Coupling biochemistry and mechanics in cell adhesion: a model for inhomogeneous stress fiber contraction. New Journal of Physics 9(11):425-425

Bielmeier C, Alt S, Weichselberger V, La Fortezza M, Harz H, Jülicher F, Salbreux G, Classen AK (2016) Interface contractility between differently fated cells drives cell elimination and cyst formation. Current Biology 26(5):563-574

Bonnet I, Marcq P, Bosveld F, Fetler L, Bellaiche Y, Graner F (2012) Mechanical state, material properties and continuous description of an epithelial tissue. J R Soc Interface 9(75):2614-23

Brodland G, Clausi D (1994) Embryonic tissue morphogenesis modeled by fem. Journal of biomechanical engineering 116(2):146-155

Brodland GW (2002) The differential interfacial tension hypothesis (dith): a comprehensive theory for the self-rearrangement of embryonic cells and tissues. Journal of biomechanical engineering 124(2):188-197

Brodland GW (2004) Computational modeling of cell sorting, tissue engulfment, and related phenomena: A review. Applied Mechanics Reviews 57(1):47-76

Brodland GW, Veldhuis JH, Kim S, Perrone M, Mashburn D, Hutson MS (2014) Cellfit: a cellular forceinference toolkit using curvilinear cell boundaries. PloS one $9(6):$ e99,116

Burgess DR (1982) Reactivation of intestinal epithelial cell brush border motility: Atp-dependent contraction via a terminal web contractile ring. The Journal of Cell Biology 95(3):853-863

Butcher JC (1987) The numerical analysis of ordinary differential equations: Runge-Kutta and general linear methods. Wiley-Interscience

Butler LC, Blanchard GB, Kabla AJ, Lawrence NJ, Welchman DP, Mahadevan L, Adams RJ, Sanson B (2009) Cell shape changes indicate a role for extrinsic tensile forces in drosophila germ-band extension. Nature cell biology 11(7):859-864

Chen HH, Brodland GW (2000) Cell-level finite element studies of viscous cells in planar aggregates. Journal of biomechanical engineering 122(4):394-401
Chen X, Brodland GW (2008) Multi-scale finite element modeling allows the mechanics of amphibian neurulation to be elucidated. Phys Biol 5(1):015,003

Collinet C, Rauzi M, Lenne PF, Lecuit T (2015) Local and tissue-scale forces drive oriented junction growth during tissue extension. Nature cell biology Paper not direclty related to work I am doing currently, but very interesting.

Colombelli J, Besser A, Kress H, Reynaud EG, Girard P, Caussinus E, Haselmann U, Small JV, Schwarz US, Stelzer EH (2009) Mechanosensing in actin stress fibers revealed by a close correlation between force and protein localization. Journal of cell science 122(10):1665-1679

Davidson LA (2012) No strings attached: new insights into epithelial morphogenesis. BMC Biology 10:105

Deguchi S, Ohashi T, Sato M (2005) Evaluation of tension in actin bundle of endothelial cells based on preexisting strain and tensile properties measurements. Molecular and Cellular Biomechanics Online 2(3):125

Deguchi S, Ohashi T, Sato M (2006) Tensile properties of single stress fibers isolated from cultured vascular smooth muscle cells. Journal of biomechanics 39(14):2603-2610

Drenckhahn D, Dermietzel R (1988) Organization of the actin filament cytoskeleton in the intestinal brush border: a quantitative and qualitative immunoelectron microscope study. The Journal of Cell Biology 107(3):1037-1048

Du X, Osterfield M, Shvartsman SY (2014) Computational analysis of three-dimensional epithelial morphogenesis using vertex models. Physical biology 11(6):066,007

Ebrahim S, Fujita T, Millis BA, Kozin E, Ma X, Kawamoto S, Baird MA, Davidson M, Yonemura S, Hisa Y (2013) Nmii forms a contractile transcellular sarcomeric network to regulate apical cell junctions and tissue geometry. Current Biology 23(8):731-736

Farhadifar R, Roper JC, Aigouy B, Eaton S, Julicher F (2007) The influence of cell mechanics, cell-cell interactions, and proliferation on epithelial packing. Current Biology 17(24):2095-104

Fletcher AG, Osterfield M, Baker RE, Shvartsman SY (2014) Vertex models of epithelial morphogenesis. Biophysical Journal 106(11):2291-304

Fristrom D, Fristrom JW (1975) The mechanis$m$ of evagination of imaginal discs of drosophila melanogaster: I. general considerations. Developmental Biology 43(1):1-23

Geiger B, Dutton AH, Tokuyasu K, Singer S (1981) Immunoelectron microscope studies of membranemicrofilament interactions: distributions of alphaactinin, tropomyosin, and vinculin in intestinal ep- 
ithelial brush border and chicken gizzard smooth muscle cells. The Journal of cell biology 91(3):614628

Glazier JA, Graner F (1993) Simulation of the differential adhesion driven rearrangement of biological cells. Physical Review E 47(3):2128

Gorfinkiel N, Blanchard GB (2011) Dynamics of actomyosin contractile activity during epithelial morphogenesis. Current Opinion in Cell Biology 23(5):531-9

Goutelle S, Maurin M, Rougier F, Barbaut X, Bourguignon L, Ducher M, Maire P (2008) The hill equation: a review of its capabilities in pharmacological modelling. Fundamental \& clinical pharmacology 22(6):633-648

Graner F, Glazier JA (1992) Simulation of biological cell sorting using a two-dimensional extended potts model. Physical review letters 69(13):2013

Graner F, Sawada Y (1993) Can surface adhesion drive cell rearrangement? part ii: a geometrical model. Journal of theoretical biology 164(4):477-506

Hannezo E, Prost J, Joanny JF (2014) Theory of epithelial sheet morphology in three dimensions. Proceedings of the National Academy of Sciences 111(1):2732

Hilgenfeldt S, Erisken S, Carthew RW (2008) Physical modeling of cell geometric order in an epithelial tissue. Proceedings of the National Academy of Sciences 105(3):907-911

Hill A (1938) The heat of shortening and the dynamic constants of muscle. Proceedings of the Royal Society of London B: Biological Sciences 126(843):136-195

Honda H, Tanemura M, Nagai T (2004) A threedimensional vertex dynamics cell model of spacefilling polyhedra simulating cell behavior in a cell aggregate. Journal of theoretical biology 226(4):439453

Honda H, Motosugi N, Nagai T, Tanemura M, Hiiragi T (2008a) Computer simulation of emerging asymmetry in the mouse blastocyst. Development 135(8):1407-14

Honda H, Nagai T, Tanemura M (2008b) Two different mechanisms of planar cell intercalation leading to tissue elongation. Developmental Dynamics $237(7): 1826-1836$

Hufnagel L, Teleman AA, Rouault H, Cohen SM, Shraiman BI (2007) On the mechanism of wing size determination in fly development. Proceedings of the National Academy of Sciences 104(10):3835-3840

Hutson MS, Veldhuis J, Ma X, Lynch HE, Cranston PG, Brodland GW (2009) Combining laser microsurgery and finite element modeling to assess cell-level epithelial mechanics. Biophysical Journal 97(12):3075-85
Ishihara S, Sugimura K, Cox S, Bonnet I, Bellaiche Y, Graner F (2013) Comparative study of non-invasive force and stress inference methods in tissue. The European Physical Journal E 36(4):1-13

Jayasinghe AK, Crews SM, Mashburn DN, Hutson MS (2013) Apical oscillations in amnioserosa cells: basolateral coupling and mechanical autonomy. Biophysical journal 105(1):255-265

Katoh K, Kano Y, Masuda M, Onishi H, Fujiwara K (1998) Isolation and contraction of the stress fiber. Molecular Biology of the Cell 9(7):1919-1938

Kaunas R, Hsu H, Deguchi S (2011) Sarcomeric model of stretch-induced stress fiber reorganization. Cell Health Cytoskeleton 3:13-22

Keller Td, Conzelman KA, Chasan R, Mooseker MS (1985) Role of myosin in terminal web contraction in isolated intestinal epithelial brush borders. The Journal of cell biology 100(5):1647-1655

Kojima H, Ishijima A, Yanagida T (1994) Direct measurement of stiffness of single actin filaments with and without tropomyosin by in vitro nanomanipulation. Proceedings of the National Academy of Sciences 91(26):12,962-12,966

Kumar S, Maxwell IZ, Heisterkamp A, Polte TR, Lele TP, Salanga M, Mazur E, Ingber DE (2006) Viscoelastic retraction of single living stress fibers and its impact on cell shape, cytoskeletal organization, and extracellular matrix mechanics. Biophysical journal 90(10):3762-3773

Landsberg KP, Farhadifar R, Ranft J, Umetsu D, Widmann TJ, Bittig T, Said A, Julicher F, Dahmann C (2009) Increased cell bond tension governs cell sorting at the drosophila anteroposterior compartment boundary. Current Biology 19(22):1950-5

Langanger G, De Mey J, Moeremans M, Daneels G, De Brabander M, Small JV (1984) Ultrastructural localization of alpha-actinin and filamin in cultured cells with the immunogold staining (igs) method. The Journal of cell biology 99(4):1324-1334

Lecuit T, Lenne PF, Munro E (2011) Force generation, transmission, and integration during cell and tissue morphogenesis. Annual review of cell and developmental biology 27:157-184

Li B, Sun SX (2014) Coherent motions in confluent cell monolayer sheets. Biophysical journal 107(7):15321541

Liu KC, Cheney RE (2012) Myosins in cell junctions. Bioarchitecture 2(5):158-170

Louveaux M, Julien JD, Mirabet V, Boudaoud A, Hamant O (2016) Cell division plane orientation based on tensile stress in arabidopsis thaliana. Proceedings of the National Academy of Sciences $p$ 201600677 
Ma X, Lynch HE, Scully PC, Hutson MS (2009) Probing embryonic tissue mechanics with laser hole drilling. Phys Biol 6(3):036,004

Major RJ, Irvine KD (2005) Influence of notch on dorsoventral compartmentalization and actin organization in the drosophila wing. Development 132(17):3823-3833

Mao Y, Baum B (2015) Tug of warthe influence of opposing physical forces on epithelial cell morphology. Developmental Biology 401(1):92-102

Mao Y, Tournier AL, Bates PA, Gale JE, Tapon N, Thompson BJ (2011) Planar polarization of the atypical myosin dachs orients cell divisions in drosophila. Genes \& development 25(2):131-136

Mao Y, Tournier AL, Hoppe A, Kester L, Thompson BJ, Tapon N (2013) Differential proliferation rates generate patterns of mechanical tension that orient tissue growth. EMBO Journal 32(21):2790-803

Matsui TS, Deguchi S, Sakamoto N, Ohashi T, Sato M (2009) A versatile micro-mechanical tester for actin stress fibers isolated from cells. Biorheology 46(5):401-15

Matsui TS, Sato M, Deguchi S (2013) High extensibility of stress fibers revealed by in vitro micromanipulation with fluorescence imaging. Biochemical and Biophysical Research Communications 434(3):444-8

Meineke FA, Potten CS, Loeffler M (2001) Cell migration and organization in the intestinal crypt using a latticefree model. Cell proliferation 34(4):253-266

Mombach JCM, de Almeida RM, Iglesias JR (1993) Mitosis and growth in biological tissues. Physical Review E 48(1):598

Mooseker MS (1985) Organization, chemistry, and assembly of the cytoskeletal apparatus of the intestinal brush border. Annual Review of Cell Biology 1(1):209-241

Murisic N, Hakim V, Kevrekidis IG, Shvartsman SY, Audoly B (2015) From discrete to continuum models of three-dimensional deformations in epithelial sheets. Biophysical journal 109(1):154-163

Nagai T, Honda H (2001) A dynamic cell model for the formation of epithelial tissues. Philosophical Magazine B 81(7):699-719

Noppe AR, Roberts AP, Gomez GA, Neufeld Z (2015) Modelling wound closure in an epithelial cell sheet using the cellular potts model. Integrative Biology $7(10): 1253-1264$

Odell GM, Oster G, Alberch P, Burnside B (1981) The mechanical basis of morphogenesis: I. epithelial folding and invagination. Developmental biology 85(2):446-462

Okuda S, Inoue Y, Eiraku M, Sasai Y, Adachi T (2013) Modeling cell proliferation for simulating three-dimensional tissue morphogenesis based on a reversible network reconnection framework. Biomechanics and modeling in mechanobiology 12(5):987996

Osterfield M, Du X, Schupbach T, Wieschaus E, Shvartsman SY (2013) Three-dimensional epithelial morphogenesis in the developing drosophila egg. Dev Cell 24(4):400-10

Owaribe K, Kodama R, Eguchi G (1981) Demonstration of contractility of circumferential actin bundles and its morphogenetic significance in pigmented epithelium in vitro and in vivo. The Journal of Cell Biology 90(2):507-514

Rauzi M, Lenne PF (2011) Cortical forces in cell shape changes and tissue morphogenesis. Current Topics in Developmental Biology 95:93

Rauzi M, Verant P, Lecuit T, Lenne PF (2008) Nature and anisotropy of cortical forces orienting drosophila tissue morphogenesis. Nat Cell Biol 10(12):1401-10

Salbreux G, Barthel LK, Raymond PA, Lubensky DK (2012) Coupling mechanical deformations and planar cell polarity to create regular patterns in the zebrafish retina. PLoS Comput Biol 8(8):e1002,618

Sandig M, Kalnins VI (1988) Subunits in zonulae adhaerentes and striations in the associated circumferential microfilament bundles in chicken retinal pigment epithelial cells in situ. Experimental cell research 175(1):1-14

Sanger JW, Sanger JM, Jockusch BM (1983) Differences in the stress fibers between fibroblasts and epithelial cells. The Journal of cell biology 96(4):961969

Schilling S, Willecke M, Aegerter-Wilmsen T, Cirpka OA, Basler K, von Mering C (2011) Cell-sorting at the $\mathrm{a} / \mathrm{p}$ boundary in the drosophila wing primordium: a computational model to consolidate observed non-local effects of hh signaling. PLoS Comput Biol 7(4):e1002,025

Smith AM, Baker RE, Kay D, Maini PK (2012) Incorporating chemical signalling factors into cell-based models of growing epithelial tissues. Journal of mathematical biology 65(3):441-463

Smutny M, Cox HL, Leerberg JM, Kovacs EM, Conti MA, Ferguson C, Hamilton NA, Parton RG, Adelstein RS, Yap AS (2010) Myosin ii isoforms identify distinct functional modules that support integrity of the epithelial zonula adherens. Nature Cell Biology 12(7):696-702

Solon J, Kaya-opur A, Colombelli J, Brunner D (2009) Pulsed forces timed by a ratchet-like mechanism drive directed tissue movement during dorsal closure. Cell 137(7):1331-1342 
Spahn P, Reuter R (2013) A vertex model of drosophila ventral furrow formation. PloS one 8(9):e75,051

Stachowiak MR, O'Shaughnessy B (2008) Kinetics of stress fibers. New Journal of Physics 10(2):025,002

Stachowiak MR, O'Shaughnessy B (2009) Recoil after severing reveals stress fiber contraction mechanisms. Biophysical journal 97(2):462-471

Staple D, Farhadifar R, Rper JC, Aigouy B, Eaton S, Jlicher F (2010) Mechanics and remodelling of cell packings in epithelia. The European Physical Journal E 33(2):117-127

Tetley RJ, Blanchard GB, Fletcher AG, Adams RJ, Sanson B (2016) Unipolar distributions of junctional myosin ii identify cell stripe boundaries that drive cell intercalation throughout drosophila axis extension. eLife 5:e12,094

Thoresen T, Lenz M, Gardel ML (2011) Reconstitution of contractile actomyosin bundles. Biophysical Journal 100(11):2698-705

Wang C, Tammi M, Guo H, Tammi R (1996) Hyaluronan distribution in the normal epithelium of esophagus, stomach, and colon and their cancers. The American journal of pathology 148(6):1861

Wang K, Ash JF, Singer S (1975) Filamin, a new highmolecular-weight protein found in smooth muscle and non-muscle cells. Proceedings of the National Academy of Sciences 72(11):4483-4486

Wartlick O, Mumcu P, Kicheva A, Bittig T, Seum C, Jlicher F, Gonzalez-Gaitan M (2011) Dynamic$\mathrm{s}$ of dpp signaling and proliferation control. Science 331(6021):1154-1159

Wehland J, Weber K (1980) Distribution of fluorescently labeled actin and tropomyosin after microinjection in living tissue culture cells as observed with tv image intensification. Experimental cell research 127(2):397-408

Weliky M, Oster G (1990) The mechanical basis of cell rearrangement. Development 109(2):373-386

Weliky M, Minsuk S, Keller R, Oster G (1991) Notochord morphogenesis in xenopus laevis: simulation of cell behavior underlying tissue convergence and extension. Development 113(4):1231-1244

Wu J, Dickinson RB, Lele TP (2012) Investigation of in vivo microtubule and stress fiber mechanics with laser ablation. Integrative Biology 4(5):471-479

Wu SK, Gomez GA, Michael M, Verma S, Cox HL, Lefevre JG, Parton RG, Hamilton NA, Neufeld Z, Yap AS (2014) Cortical f-actin stabilization generates apicallateral patterns of junctional contractility that integrate cells into epithelia. Nature Cell Biology 16(2):167-178

Yonemura S, Itoh M, Nagafuchi A, Tsukita S (1995) Cell-to-cell adherens junction formation and actin fil- ament organization: similarities and differences between non-polarized fibroblasts and polarized epithelial cells. Journal of cell science 108(1):127-142 


\section{University Library}

\section{- M M N E R VA A gateway to Melbourne's research publications}

Minerva Access is the Institutional Repository of The University of Melbourne

Author/s:

Boyd, ARB;Moore, S;Sader, JE;Lee, PVS

Title:

Modelling apical columnar epithelium mechanics from circumferential contractile fibres

Date:

2017-10-01

Citation:

Boyd, A. R. B., Moore, S., Sader, J. E. \& Lee, P. V. S. (2017). Modelling apical columnar epithelium mechanics from circumferential contractile fibres. BIOMECHANICS AND MODELING IN MECHANOBIOLOGY, 16 (5), pp.1555-1568. https://doi.org/10.1007/ s10237-017-0905-7.

Persistent Link:

http://hdl.handle.net/11343/282897 